\title{
LINEAR DIFFERENTIAL EQUATIONS WITH SOLUTIONS IN THE GROWTH SPACE $\boldsymbol{H}_{\omega}^{\infty}$
}

\author{
Juha-Matti Huusko, Taneli Korhonen and Atte Reijonen \\ University of Eastern Finland, Department of Physics and Mathematics \\ P.O. Box 111, 80101 Joensuu, Finland; juha-matti.huusko@uef.fi \\ University of Eastern Finland, Department of Physics and Mathematics \\ P.O. Box 111, 80101 Joensuu, Finland; taneli.korhonen@uef.fi \\ University of Eastern Finland, Department of Physics and Mathematics \\ P.O. Box 111, 80101 Joensuu, Finland; atte.reijonen@uef.fi
}

$$
\begin{aligned}
& \text { Abstract. Sufficient conditions for solutions of } \\
& \qquad f^{(n)}+A_{n-1}(z) f^{(n-1)}+\cdots+A_{1}(z) f^{\prime}+A_{0}(z) f=A_{n}(z)
\end{aligned}
$$

and their derivatives to be in $H_{\omega}^{\infty}(D)$ are given by limiting the growth of coefficients $A_{0}(z), \ldots A_{n}(z)$. Here $H_{\omega}^{\infty}(D)$ consists of those analytic functions $f$ in a domain $D$ for which $|f(z)| \omega(z)$ is uniformly bounded. In particular, the case where $D$ is the unit disc is considered. The theorems obtained generalize and improve certain results in the literature. Moreover, by using one of the main results, one can give a straightforward proof of a classical result regarding the situation where the coefficients are polynomials.

\section{Introduction}

We study the growth of solutions of the differential equation

$$
f^{(n)}+A_{n-1}(z) f^{(n-1)}+\cdots+A_{1}(z) f^{\prime}+A_{0}(z) f=A_{n}(z), \quad n \geq 2,
$$

where $A_{0}(z), \ldots, A_{n}(z)$ are analytic in a domain $D$ of the complex plane $\mathbf{C}$, denoted by $A_{0}, \ldots, A_{n} \in \mathcal{H}(D)$ for short. In particular, we consider the case where $D$ is the unit disc $\mathbf{D}=\{z \in \mathbf{C}:|z|<1\}$. Hence, for simplicity, notations mentioned below are defined for $\mathbf{D}$ but on request we use their analogies also for other domains.

Our main purpose is to find conditions which guarantee that all solutions of (1) or their derivatives belong to a growth space

$$
H_{\omega}^{\infty}=\left\{g \in \mathcal{H}(\mathbf{D}):\|g\|_{H_{\omega}^{\infty}}:=\sup _{z \in \mathbf{D}}|g(z)| \omega(z)<\infty\right\} .
$$

Here $\omega$ is a weight, which means that $\omega: \mathbf{D} \rightarrow(0, \infty)$ is bounded and measurable. In the case where $\omega(z)=\omega(|z|)$ for all $z \in \mathbf{D}$, we say that $\omega$ is radial. If $\omega(z)=(1-|z|)^{p}$ with $p \in(0, \infty)$, we write $H_{\omega}^{\infty}=H_{p}^{\infty}$. Also, the question of when all solutions belong to the $\alpha$-Bloch space $\mathcal{B}^{\alpha}$ with $\alpha \in(0, \infty)$, which consists of $g \in \mathcal{H}(\mathbf{D})$ such that $\|g\|_{\mathcal{B}^{\alpha}}:=\sup _{z \in \mathbf{D}}\left|g^{\prime}(z)\right|(1-|z|)^{\alpha}<\infty$, is considered. Note that if $\alpha=1$, then $\mathcal{B}^{\alpha}$ is the classical Bloch space $\mathcal{B}$.

doi:10.5186/aasfm.2016.4128

2010 Mathematics Subject Classification: Primary 34M10; Secondary 30H30.

Key words: Differential equation, unit disc, Bloch space, growth space.

This research was supported in part by the Academy of Finland, Project \#268009, and the Faculty of Science and Forestry of the University of Eastern Finland, Project \#930349. The third author was also supported by the Väisälä Foundation. 
The growth of fast growing solutions of (1) is typically measured in terms of the Nevanlinna characteristic function [9]. For slowly growing solutions some other methods may give better results. Some useful techniques are, for example, Gronwall's lemma [7], Herold's comparison theorem [11], Picard's successive approximations $[2,5]$ and methods based on Carleson measures [10, 13, 14, 15]. Moreover, in the case of the complex plane, Wiman-Valiron theory is a commonly used method [12]. We do not use any of these methods; instead, our calculations are based on straightforward integral estimates.

It is well known that the growth of the coefficients $A_{0}(z), \ldots, A_{n}(z)$ of $(1)$ restricts the growth of solutions. For example, if the coefficients grow slow enough, then all solutions are bounded, while if the coefficients grow fast enough, then the solutions may grow faster than any pre-given function. Therefore, if one wants to force all solutions to $H_{\omega}^{\infty}$, it suffices to give a strong enough growth condition for the coefficients; for example, one can require that the norms $\left\|A_{0}\right\|_{H_{n}^{\infty}}, \ldots,\left\|A_{n-1}\right\|_{H_{1}^{\infty}}$ are small enough. This condition can be found by applying the differential equation and using suitable integral estimates.

Using the integral estimates method mentioned above with a condition on the norms $\left\|A_{0}\right\|_{H_{n}^{\infty}}, \ldots,\left\|A_{n-1}\right\|_{H_{1}^{\infty}}$, we prove Theorem 1. This result generalizes and improves [10, Theorems 3.1 and 3.3]. Moreover, as a special case, we also give a solution to the following problem due to the late Danikas, which has been open since the 1997 summer school "Function Spaces and Complex Analysis" held at the Mekrijärvi Research Station in Ilomantsi, Finland: Give a condition for $A(z)$ such that all solutions of

$$
f^{\prime \prime}+A(z) f=0
$$

belong to the Bloch space $\mathcal{B}$. More precisely, Theorem 1 yields that if $\sup _{z \in \mathbf{D}}[-|A(z)|$ $\left.(1-|z|)^{2} \log (1-|z|)\right]<1$, then all solutions of $(2)$ belong to $\mathcal{B}$. This particular result is sharp in the sense that the assumption cannot be relaxed to $\sup _{z \in \mathbf{D}}[-|A(z)|(1-$ $\left.|z|)^{2} \log (1-|z|)\right]<1+\varepsilon$ for any $\varepsilon \in(0, \infty)$. It is worth noticing that all previous results known to the authors, including those given in $[10,13]$, force the solutions to some proper subspace of $\mathcal{B}$ and hence form only a partial solution to the problem.

Our second main result, Theorem 2, is proved by applying an integral condition, instead of radial growth space conditions, for the coefficients. In this case, the result is valid also in other domains than just the unit disc. As a consequence of the result, an alternative version of Theorem 1 is verified. An application for polynomial coefficients is also obtained.

A classical result [18, Satz 1] of Wittich states that every solution of (1), where the coefficients $A_{0}(z), \ldots, A_{n-1}(z)$ are entire and $A_{n} \equiv 0$, has a finite order of growth if and only if all coefficients $A_{0}(z), \ldots, A_{n-1}(z)$ are polynomials. Moreover, if the coefficients are polynomials, then the order of growth $\sigma(f)$ of any solution $f$ satisfies the well-known estimate

$$
\sigma(f):=\limsup _{r \rightarrow \infty} \frac{\log \log M(r, f)}{\log r} \leq \max _{0 \leq j \leq n-1}\left\{1+\frac{\operatorname{deg}\left(A_{j}\right)}{n-j}\right\},
$$

where $M(r, f)$ is the maximum modulus of $f$ on the circle of radius $r$ centered at the origin. This estimate can be proved in a straightforward manner without any heavy machinery by using Theorem 2; see Section 4 . In the literature, one can find more technical proofs based on, for example, Wiman-Valiron theory [12, Theorem 8.3] and Herold's comparison theorem [8, p. 244]. By applying Gronwall's lemma or Picard's 
successive approximations, only a weaker version of the estimate has been proved $[5,8]$.

The remainder of this paper is organized as follows: In the next section, we introduce our main results, Theorems 1 and 2. We also prove an alternative version of Theorem 1 by using Theorem 2. The main purpose of Section 3 is to improve results of [13]. More precisely, we improve [13, Theorems 2.1 and 2.6] in the case where the nondecreasing function $K$ is continuous, and also give a partial improvement of [13, Theorem 2.4]. In addition to this, we discuss the sharpness of our main results. Section 4 contains a simple proof of the essential part of [12, Theorem 8.3] which concerns a differential equation with polynomial coefficients in the plane. Sections 5 and 6 contain the proofs of Theorems 1 and 2, respectively.

\section{Main results}

In this section, we present our main results, Theorems 1 and 2 . We start by introducing conditions and notations needed in the statement of Theorem 1.

In Theorem 1, we require that the radial weight $\omega: \mathbf{D} \rightarrow(0, \infty)$ satisfies the conditions

$$
\limsup _{r \rightarrow 1^{-}} \omega(r) \int_{0}^{r} \frac{d s}{\omega(s)(1-s)}<M<\infty,
$$

for some $M=M(\omega) \in(0, \infty)$, and

$$
\limsup _{r \rightarrow 1^{-}} \frac{\omega(r)}{\omega\left(\frac{1+\varepsilon r}{1+\varepsilon}\right)}<m
$$

for some constants $\varepsilon \in(0, \infty)$ and $m=m(\omega, \varepsilon) \in(0, \infty)$. It should be noted that (3) implies that there exists $M_{k}=M_{k}(\omega, k) \in(0, M]$ and $M_{0}=M_{0}(\omega) \in(0, \infty)$ such that

$$
\limsup _{r \rightarrow 1^{-}} \omega(r)(1-r)^{k-1} \int_{0}^{r} \frac{d s}{\omega(s)(1-s)^{k}}<M_{k}, \quad k=1, \ldots, n
$$

and

$$
\omega(t) \int_{0}^{t} \frac{d s}{\omega(s)(1-s)}<M_{0}, \quad t \in(0,1) .
$$

The conditions (3) and (4) play key roles in Theorem 1. Hence, before stating the theorem, we list some observations about (3) and (4).

(i) The conditions (3) and (4) are independent. For example, $\omega(r)=\exp \left(-\frac{1}{1-r}\right)$ satisfies (3) but fails (4). On the other hand, $\omega(r)=\left(\log \frac{e}{1-r}\right)^{-1}$ satisfies (4) but fails (3).

(ii) If $\omega$ satisfies (3), then there exists $p=p(\omega) \in(0, \infty)$ such that $\omega(r) /(1-r)^{p}$ is bounded [17, Lemma 2].

(iii) It is possible that (4) holds for some $\varepsilon$ but not for all. For example,

$$
\omega(r)=(1-r) \sin ^{2}\left(\frac{\pi \log \frac{1}{1-r}}{\log 2}\right)+(1-r)^{2} \cos ^{2}\left(\frac{\pi \log \frac{1}{1-r}}{\log 2}\right)
$$

satisfies (4) for $\varepsilon=1$ but not for $\varepsilon=\pi$. However, if (4) holds for some $\varepsilon$, then it holds for some arbitrarily small $\varepsilon$. 
(iv) If $\omega$ is nonincreasing and (4) holds for some $\varepsilon$, then it holds for all $\varepsilon$. Hence, in this case, (4) is equivalent to the doubling condition $\omega(r) \leq m \omega\left(\frac{1+r}{2}\right)$ when $r \in[0,1)$ is close enough to one.

(v) If the condition (4) is valid, then, in (3), the factor $1-s$ is in a certain sense the best possible. Namely, if $\nu:(0,1) \rightarrow(0, \infty), \omega$ satisfies (4) for some $\varepsilon, m \in(0, \infty), \omega \nu$ is nonincreasing and there exists $M=M(\omega, \nu) \in(0, \infty)$ such that

$$
\limsup _{t \rightarrow 1^{-}} \omega(t) \int_{0}^{t} \frac{d s}{\omega(s) \nu(s)}<M
$$

then we have

$$
M>\omega\left(\frac{1+\varepsilon r}{1+\varepsilon}\right) \int_{r}^{\frac{1+\varepsilon r}{1+\varepsilon}} \frac{d s}{\omega(s) \nu(s)} \geq \omega\left(\frac{1+\varepsilon r}{1+\varepsilon}\right) \frac{\left(\frac{1+\varepsilon r}{1+\varepsilon}-r\right)}{\omega(r) \nu(r)}>\frac{1}{m} \frac{1}{1+\varepsilon} \frac{1-r}{\nu(r)}
$$

for sufficiently large $r \in[0,1)$. In particular, $(1-r) / \nu(r)$ is bounded if $r$ is close enough to one.

Next we state Theorem 1 , in which we use the notation $\omega_{p}(z)=\omega(z)(1-|z|)^{p}$, where $\omega$ is a radial weight and $p \in \mathbf{R}$.

Theorem 1. Let $\omega$ be a radial weight in the unit disc satisfying (3) and (4). Then the following assertions hold:

(a) If $A_{n} \in H_{\omega_{n}}^{\infty}$ and

$$
E:=P_{n}\left(\left\|A_{0}\right\|_{H_{n}^{\infty}}+m \sum_{k=1}^{n-1} k !(1+\varepsilon)^{k}\left\|A_{k}\right\|_{H_{n-k}^{\infty}}\right)<1,
$$

where $P_{n}=\prod_{k=1}^{n} M_{k}$ with constants $M_{k}$ as in (5) and $m, \varepsilon$ are as in (4), then all solutions of (1) belong to $H_{\omega}^{\infty}$.

(b) If $A_{n} \in H_{\omega_{n-1}}^{\infty}$ and

$$
\begin{aligned}
F:= & P_{n-1}\left(\sup _{z \in \mathbf{D}}\left|A_{0}(z)\right| \omega(z)(1-|z|)^{n-1} \int_{0}^{|z|} \frac{d r}{\omega(r)}\right. \\
& \left.+\left\|A_{1}\right\|_{H_{n-1}^{\infty}}+m \sum_{k=1}^{n-2} k !(1+\varepsilon)^{k}\left\|A_{k+1}\right\|_{H_{n-k-1}^{\infty}}\right)<1,
\end{aligned}
$$

where $P_{n-1}=\prod_{k=1}^{n-1} M_{k}$ with constants $M_{k}$ as in (5) and $m, \varepsilon$ are as in (4), then the derivative of every solution of (1) belongs to $H_{\omega}^{\infty}$.

Moreover, if we consider the equations

$$
f^{(n)}+A_{0}(z) f=0 \quad \text { and } \quad f^{(n)}+A_{1}(z) f^{\prime}+A_{0}(z) f=0
$$

in (a) and (b), respectively, then the assumption (4) regarding $\omega$ is not necessary.

In what follows, we present another result where, instead of considering the norms $\left\|A_{0}\right\|_{H_{n}^{\infty}}, \ldots,\left\|A_{n-1}\right\|_{H_{1}^{\infty}}$, we establish an integral condition on the coefficients and their derivatives. This result is also more general in the sense that the weight $\omega$ does not need to be radial and the unit disc $\mathbf{D}$ may be replaced by some other domain.

We call a domain $D$ on the complex plane starlike if $0 \in D$ and, for each point $z \in D$, the line segment from the origin to $z$ is contained in $D$. For a weight $\omega$ 
(not necessarily radial) in such a domain $D$ and functions $A_{0}, A_{1}, \ldots, A_{n-1} \in \mathcal{H}(D)$, denote

$$
I_{1, \omega}(z)=I_{1, \omega}^{*}(z)=\int_{0}^{z} \frac{\left|A_{n-1}(\xi)\right|}{\omega(\xi)}|d \xi|
$$

and

$$
I_{m, \omega}(z)=\int_{0}^{z} \int_{0}^{\xi_{1}} \cdots \int_{0}^{\xi_{m-1}}\left|\sum_{j=1}^{m}(-1)^{m-j}\left(\begin{array}{c}
n-j \\
m-j
\end{array}\right) A_{n-j}^{(m-j)}\left(\xi_{m}\right)\right| \frac{\left|d \xi_{m}\right| \cdots\left|d \xi_{1}\right|}{\omega\left(\xi_{m}\right)}, \quad z \in D
$$

for $m=2, \ldots, n$ and

$$
I_{m, \omega}^{*}(z)=\int_{0}^{z} \cdots \int_{0}^{\xi_{m-1}}\left|\sum_{j=1}^{m}(-1)^{m-j}\left(\begin{array}{c}
n-1-j \\
m-j
\end{array}\right) A_{n-j}^{(m-j)}\left(\xi_{m}\right)\right| \frac{\left|d \xi_{m}\right| \cdots\left|d \xi_{1}\right|}{\omega\left(\xi_{m}\right)}, \quad z \in D,
$$

for $m=2, \ldots, n-1$, where the integration paths are line segments. With these concepts and notations established, we give the following result.

Theorem 2. Let $D$ be a starlike domain and let $\omega: D \rightarrow(0, \infty)$ be a weight. Then the following assertions hold:

(a) If

$$
E:=\sup _{z \in D} \omega(z) \sum_{m=1}^{n} I_{m, \omega}(z)<1
$$

and the function $z \mapsto \int_{0}^{z} \int_{0}^{\xi_{1}} \cdots \int_{0}^{\xi_{n-1}} A_{n}\left(\xi_{n}\right) d \xi_{n} \cdots d \xi_{1}$ belongs to $H_{\omega}^{\infty}(D)$, (b) If then all solutions of (1) belong to $H_{\omega}^{\infty}(D)$.

$$
\begin{aligned}
F:= & \sup _{z \in D} \omega(z)\left[\int_{0}^{z} \cdots \int_{0}^{\xi_{n-2}}\left|A_{0}\left(\xi_{n-1}\right)\right| \int_{0}^{\xi_{n-1}} \frac{\left|d \xi_{n}\right|}{\omega\left(\xi_{n}\right)}\left|d \xi_{n-1}\right| \cdots\left|d \xi_{1}\right|\right. \\
& \left.+\sum_{m=1}^{n-1} I_{m, \omega}^{*}(z)\right]<1
\end{aligned}
$$

and the function $z \mapsto \int_{0}^{z} \int_{0}^{\xi_{1}} \cdots \int_{0}^{\xi_{n-2}} A_{n}\left(\xi_{n-1}\right) d \xi_{n-1} \cdots d \xi_{1}$ belongs to $H_{\omega}^{\infty}(D)$, then the derivative of every solution of (1) belongs to $H_{\omega}^{\infty}(D)$.

Note that the conditions (7) and (8) both imply that $\omega$ needs to be bounded, unless all the coefficients $A_{0}(z), \ldots, A_{n-1}(z)$ are identically zero.

It is worth noticing that the method used to prove Theorem 2 works also in more general domains than just those which are starlike with respect to the origin. In fact, if one chooses the paths of integration and the compact sets $K$ appropriately, the method may be used in any domain $D$. For example, let $D \subsetneq \mathbf{C}$ be any simply connected domain and let $\phi: \mathbf{D} \rightarrow D$ be a Riemann map from $\mathbf{D}$ onto $D$. Then choosing the paths of integration in the proof of Theorem 2 to be $l_{z}=\phi\left(\left[0, \phi^{-1}(z)\right]\right)$, for $z \in D$, and taking the compact sets as $K=\bigcup_{z \in K_{0}} \phi\left(\left[0, \phi^{-1}(z)\right]\right)$, where $K_{0}$ is an arbitrary compact subset of $D$, one sees that the following result holds:

If the function $z \mapsto \int_{l_{z}} \int_{l_{\xi_{1}}} \cdots \int_{l_{\xi_{n-1}}} A_{n}\left(\xi_{n}\right) d \xi_{n} \cdots d \xi_{1}$ belongs to $H_{\omega}^{\infty}(D)$ and

$$
\sup _{z \in D} \omega(z) \sum_{m=1}^{n} I_{m, \omega}^{(\phi)}(z)<1
$$


where

$$
I_{1, \omega}^{(\phi)}(z)=\int_{l_{z}} \frac{\left|A_{n-1}(\xi)\right|}{\omega(\xi)}|d \xi|=\int_{0}^{1} \frac{\left|A_{n-1}\left(\phi\left(t \phi^{-1}(z)\right)\right)\right|}{\omega\left(\phi\left(t \phi^{-1}(z)\right)\right)}\left|\phi^{\prime}\left(t \phi^{-1}(z)\right) \phi^{-1}(z)\right| d t, \quad z \in D,
$$

and

$$
I_{m, \omega}^{(\phi)}(z)=\int_{l_{z}} \int_{l_{\xi_{1}}} \ldots \int_{l_{\xi_{m-1}}}\left|\sum_{j=1}^{m}(-1)^{m-j}\left(\begin{array}{c}
n-j \\
m-j
\end{array}\right) A_{n-j}^{(m-j)}\left(\xi_{m}\right)\right| \frac{\left|d \xi_{m}\right| \cdots\left|d \xi_{1}\right|}{\omega\left(\xi_{m}\right)}, \quad z \in D,
$$

for $m=2, \ldots, n$, then all solutions of (1) belong to $H_{\omega}^{\infty}(D)$.

Even in this rather simple example, it is clear that the choices of integration paths and compact sets done above are not the only nor necessarily the best ones. However, the example above is an easy way to illustrate the generality of the argument used in the proof of Theorem 2. It is also a way to pinpoint the connection between the choice of the paths of integration and that of compact sets: The compact sets need to contain all the integration paths from the chosen fixed point $z_{0} \in D$ (in the above example $\left.z_{0}=\phi(0)\right)$ to other points in the compact set. Hence one also cannot choose the paths of integration randomly but some kind of systematic approach or control over the paths is required.

Finally, we derive a result of the same nature as Theorem 1 from Theorem 2 . The main difference is that the result is not as sharp as Theorem 1 but the weight $\omega$ does not need to satisfy the condition (4).

Theorem 3. Let $\omega$ be a radial weight in the unit disc satisfying (3). Then the following assertions hold:

(a) There exists $\alpha=\alpha(\omega, n) \in(0, \infty)$ such that if

$$
\left\|A_{j}\right\|_{H_{n-j}^{\infty}} \leq \alpha, \quad j=0, \ldots, n-1
$$

and the function $z \mapsto \int_{0}^{z} \int_{0}^{\xi_{1}} \cdots \int_{0}^{\xi_{n-1}} A_{n}\left(\xi_{n}\right) d \xi_{n} \cdots d \xi_{1}$ belongs to $H_{\omega}^{\infty}$, then all solutions of (1) belong to $H_{\omega}^{\infty}$.

(b) There exists $\alpha=\alpha(\omega, n) \in(0, \infty)$ such that if

$$
\begin{aligned}
& \left\|A_{j}\right\|_{H_{n-j}^{\infty}} \leq \alpha, \quad j=1, \ldots, n-1 \\
& \sup _{z \in D} \omega(z)\left[\int_{0}^{z} \cdots \int_{0}^{\xi_{n-2}}\left|A_{0}\left(\xi_{n-1}\right)\right| \int_{0}^{\xi_{n-1}} \frac{\left|d \xi_{n}\right|}{\omega\left(\xi_{n}\right)}\left|d \xi_{n-1}\right| \cdots\left|d \xi_{1}\right|\right]<1
\end{aligned}
$$

and the function $z \mapsto \int_{0}^{z} \int_{0}^{\xi_{1}} \cdots \int_{0}^{\xi_{n-2}} A_{n}\left(\xi_{n-1}\right) d \xi_{n-1} \cdots d \xi_{1}$ belongs to $H_{\omega}^{\infty}$, then the derivative of every solution of (1) belongs to $H_{\omega}^{\infty}$.

Proof. By (6), we obtain

$$
\begin{aligned}
& I_{m, \omega}(z) \omega(z)=\omega(z) \int_{0}^{z} \int_{0}^{\xi_{1}} \cdots \int_{0}^{\xi_{m-1}}\left|\sum_{j=1}^{m}(-1)^{m-j}\left(\begin{array}{c}
n-j \\
m-j
\end{array}\right) A_{n-j}^{(m-j)}\left(\xi_{m}\right)\right| \frac{\left|d \xi_{m}\right| \cdots\left|d \xi_{1}\right|}{\omega\left(\xi_{m}\right)} \\
& \leq C \omega(z) \int_{0}^{z} \int_{0}^{\xi_{1}} \cdots \int_{0}^{\xi_{m-1}} \sum_{j=1}^{m}\left|A_{n-j}^{(m-j)}\left(\xi_{m}\right)\right| \frac{\left|d \xi_{m}\right| \cdots\left|d \xi_{1}\right|}{\omega\left(\xi_{m}\right)} \\
& \leq C \sum_{j=1}^{m} \sup _{|\xi| \leq|z|}\left|A_{n-j}^{(m-j)}(\xi)\right|(1-|\xi|)^{m} \omega(z) \int_{0}^{z} \int_{0}^{\xi_{1}} \cdots \int_{0}^{\xi_{m-1}} \frac{\left|d \xi_{m}\right| \cdots\left|d \xi_{1}\right|}{\omega\left(\xi_{m}\right)\left(1-\left|\xi_{m}\right|\right)^{m}} \\
& \leq C^{\prime} \sum_{j=1}^{m} \sup _{|\xi| \leq|z|}\left|A_{n-j}^{(m-j)}(\xi)\right|(1-|\xi|)^{m}, \quad z \in \mathbf{D},
\end{aligned}
$$


for some constants $C \in(0, \infty)$ and $C^{\prime}=C^{\prime}(\omega, n) \in(0, \infty)$. Therefore Lemma 10 for classical weights yields

$$
\begin{aligned}
\sup _{|\xi| \leq|z|} \omega(\xi) \sum_{m=1}^{n} I_{m, \omega}(\xi) & \leq \sum_{m=1}^{n} \sup _{|\xi| \leq|z|} \omega(\xi) I_{m, \omega}(\xi) \\
& \leq C^{\prime} \sum_{m=1}^{n} \sum_{j=1}^{m} \sup _{|\xi| \leq|z|}\left|A_{n-j}^{(m-j)}(\xi)\right|(1-|\xi|)^{m} \\
& \leq n C^{\prime \prime} \sum_{j=0}^{n-1} \sup _{|\xi| \leq \rho}\left|A_{j}(\xi)\right|(1-|\xi|)^{n-j}
\end{aligned}
$$

where $\rho=(1+|z|) / 2$ and $C^{\prime \prime}=C^{\prime \prime}(\omega, n) \in(0, \infty)$. Now, we have

$$
E=\sup _{z \in \mathbf{D}} \omega(z) \sum_{m=1}^{n} I_{m, \omega}(z) \leq n C^{\prime \prime} \sum_{j=0}^{n-1}\left\|A_{j}\right\|_{H_{n-j}^{\infty}}<1
$$

for $\left\|A_{j}\right\|_{H_{n-j}^{\infty}}<\frac{1}{n^{2} C^{\prime \prime}}$ with all indices $j=0, \ldots, n-1$. Hence the assertion (a) follows by Theorem 2 . The assertion (b) can be proved in a similar manner.

\section{Solutions in $\mathcal{B}^{\alpha}, Q_{K}$ or $Q_{K, 0}$}

We begin this section by stating a version of Theorem 1 where $\omega(r)=(1-r)^{p}$ with $p \in(0, \infty)$. After that we discuss the sharpness of Theorems 1 and 2 and some of their consequences. In particular, consequences of Theorem 1, related to the cases where all solutions of differential equations belong to $\mathcal{B}^{\alpha}, Q_{K}$ or $Q_{K, 0}$, are stated.

Corollary 4. Let $f$ be a solution of the equation (1) with $A_{n} \equiv 0$. Then the following assertions hold:

(a) If, for $p \in(0, \infty)$,

$$
E:=\prod_{j=1}^{n} \frac{1}{p+j-1}\left(\left\|A_{0}\right\|_{H_{n}^{\infty}}+\sum_{k=1}^{n-1} k ! \frac{(k+p)^{k+p}}{k^{k} p^{p}}\left\|A_{k}\right\|_{H_{n-k}^{\infty}}\right)<1,
$$

then

$$
\|f\|_{H_{p}^{\infty}} \leq \frac{|f(0)|+\sum_{k=1}^{n-1} \prod_{j=1}^{k} \frac{1}{p+j-1}\left|f^{(k)}(0)\right|}{1-E} .
$$

(b) If, for $\alpha \in(0, \infty)$,

$$
\begin{aligned}
F:= & \prod_{j=1}^{n-1} \frac{1}{\alpha+j-1}\left(\sup _{z \in \mathbf{D}}\left|A_{0}(z)\right|(1-|z|)^{\alpha+n-1} \int_{0}^{|z|} \frac{d r}{(1-r)^{\alpha}}\right. \\
& \left.+\left\|A_{1}\right\|_{H_{n-1}^{\infty}}+\sum_{k=1}^{n-2} k ! \frac{(k+\alpha)^{k+\alpha}}{k^{k} \alpha^{\alpha}}\left\|A_{k+1}\right\|_{H_{n-k-1}^{\infty}}\right)<1,
\end{aligned}
$$

then

$$
\|f\|_{\mathcal{B}^{\alpha}} \leq \frac{\prod_{j=1}^{n-1} \frac{1}{\alpha+j-1}\left\|A_{0}\right\|_{H_{\alpha+n-1}^{\infty}}|f(0)|+\left|f^{\prime}(0)\right|+\sum_{k=2}^{n-1} \prod_{j=1}^{k-1} \frac{1}{\alpha+j-1}\left|f^{(k)}(0)\right|}{1-F}
$$

The following example shows that, in the case of equation (2), Theorem 2 and Corollary 4, hence also Theorem 1, are sharp in the sense that we cannot replace the assumption $E<1$ or $F<1$ by $E<1+\varepsilon$ or $F<1+\varepsilon$, respectively, for any $\varepsilon \in(0, \infty)$. 
Example 5. Let us consider the equation (2).

(a) If $A(z)=-(p+\alpha)(p+\alpha+1)(1-z)^{-2}$ for $p \in(0, \infty)$ and $\alpha \in[0, \infty)$, then (2) has a solution base $\left\{f_{1}, f_{2}\right\}$, where

$$
f_{1}(z)=(1-z)^{-p-\alpha} \text { and } f_{2}(z)=(1-z)^{p+\alpha+1} .
$$

Hence, if $\alpha=0$, then all solutions belong to $H_{p}^{\infty}$ and $E=1$ in Theorem 2(a) and Corollary 4(a). On the other hand, for any $\varepsilon \in(0, \infty)$, we find $\alpha=$ $\alpha(\varepsilon) \in(0, \infty)$ such that $f_{1} \notin H_{p}^{\infty}$ and $E \in(1,1+\varepsilon)$ in these results.

(b) If $A(z)=-\alpha(1-z)^{-2}\left((\alpha-1)\left(\log \frac{e}{1-z}\right)^{-2}+\left(\log \frac{e}{1-z}\right)^{-1}\right)$ for $\alpha \in[1, \infty)$, then (2) has a solution base $\left\{f_{1}, f_{2}\right\}$, where

$$
f_{1}(z)=\left(\log \frac{e}{1-z}\right)^{\alpha} \text { and } f_{2}(z)=\left(\log \frac{e}{1-z}\right)^{\alpha} \int_{0}^{z}\left(\log \frac{e}{1-\zeta}\right)^{-2 \alpha} d \zeta \text {. }
$$

Here

$$
\left|\int_{0}^{z}\left(\log \frac{e}{1-\zeta}\right)^{-2 \alpha} d \zeta\right| \leq\left(\log \frac{e}{2}\right)^{-2 \alpha}
$$

and

$$
\left|f_{2}^{\prime}(z)\right| \leq\left(\log \frac{e}{2}\right)^{-\alpha}\left|f_{1}^{\prime}(z)\right|+\left(\log \frac{e}{2}\right)^{-2 \alpha}
$$

for $z \in \mathbf{D}$. Hence, if $\alpha=1$, then all solutions belong to $\mathcal{B}$ and $F=1$ in Theorem 2(b) and Corollary 4(b). On the other hand, for any $\varepsilon \in(0, \infty)$, we find $\alpha=\alpha(\varepsilon) \in(1, \infty)$ such that $f_{1} \notin \mathcal{B}$ and $F \in(1,1+\varepsilon)$ in these results.

Next we turn our attention to $Q_{K}$ and $Q_{K, 0}$ spaces. In particular, our purpose is to improve results in [13].

Let $Q_{K}$ be the space of functions $f \in \mathcal{H}(\mathbf{D})$ such that

$$
\sup _{a \in \mathbf{D}} \int_{\mathbf{D}}\left|f^{\prime}(z)\right|^{2} K(g(z, a)) d m(z)<\infty,
$$

where $K:[0, \infty) \rightarrow[0, \infty)$ is nondecreasing, $g(z, w)=\log \left|\frac{1-\bar{w} z}{w-z}\right|$ is Green's function and $d m(z)$ is the Lebesgue area measure. Respectively, $Q_{K, 0}$ is the space of functions $f \in \mathcal{H}(\mathbf{D})$ such that

$$
\lim _{|a| \rightarrow 1^{-}} \int_{\mathbf{D}}\left|f^{\prime}(z)\right|^{2} K(g(z, a)) d m(z)=0 .
$$

If $K \equiv 1$, then $Q_{K}$ is the Dirichlet space $\mathcal{D}$.

For the next result, we introduce some properties of $Q_{K}$ and $Q_{K, 0}$. We begin by introducing a standard assumption which guarantees that $Q_{K}$ contains non-constant functions.

(i) If

$$
\int_{1}^{\infty} K(r) e^{-2 r} d r<\infty
$$

does not hold, then $Q_{K}$ contains constant functions only.

In the future, we assume that $K:[0, \infty) \rightarrow[0, \infty)$ is continuous, nondecreasing and satisfies (10). Then the following facts are true:

(ii) The inclusion $Q_{K} \subset \mathcal{B}$ is always valid. Moreover, $Q_{K}=\mathcal{B}$ if and only if

$$
\int_{0}^{1} \frac{K(-\log r)}{(1-r)^{2}} r d r<\infty
$$


(iii) The inclusion $\mathcal{D} \subset Q_{K}$ is always valid. Moreover, $\mathcal{D}=Q_{K}$ if and only if $K(0)>0$, while $\mathcal{D} \subset Q_{K, 0}$ if and only if $K(0)=0$.

(iv) For $\alpha \in\left[\frac{1}{2}, 1\right)$, the conditions $\mathcal{B}^{\alpha} \subset Q_{K, 0}, \mathcal{B}^{\alpha} \subset Q_{K}$ and

$$
\int_{0}^{1} \frac{K(-\log r)}{(1-r)^{2 \alpha}} r d r<\infty
$$

are equivalent.

(v) If $K(r)=r^{p}$ for $p \in(0, \infty)$, then $Q_{K}$ is the classical $Q^{p}$ space.

Proofs of the facts (i)-(v) and more details about $Q_{K}$ spaces can be found in [4].

Now, by using the facts (i)-(iv) and the trivial inclusion $\mathcal{B}^{\alpha} \subset \mathcal{D}$ for $\alpha \in\left(0, \frac{1}{2}\right)$, we obtain the following consequence of Corollary 4.

Corollary 6. Let $f$ be a solution of the equation (1) with $A_{n} \equiv 0$. Then the following assertions hold:

(a) If (9) with $\alpha=1$ and (11) hold, then $f \in \mathcal{B}=Q_{K}$.

(b) If (9) with $\alpha \in\left[\frac{1}{2}, 1\right)$ and (12) hold, then $f \in \mathcal{B}^{\alpha} \subset Q_{K, 0}$.

(c) If (9) holds with $\alpha \in\left(0, \frac{1}{2}\right)$, then $f \in \mathcal{B}^{\alpha} \subset \mathcal{D} \subset Q_{K}$. Moreover, $f \in \mathcal{B}^{\alpha} \subset$ $\mathcal{D} \subset Q_{K, 0}$ if $K(0)=0$.

It is worth noticing that Corollary 6(c) improves [13, Theorems 2.1 and 2.6] in the case where the nondecreasing function $K$ is also continuous. In particular, the condition concerning the coefficient $A_{0}(z)$ is weaker in Corollary 6(c). Namely, in Corollary $6(\mathrm{c})$, we only have to assume that $\left\|A_{0}\right\|_{H_{n-1 / 2-\varepsilon}^{\infty}}$ is sufficiently small for some $\varepsilon \in(0, \infty)$, whereas in [13, Theorem 2.1] or [13, Theorem 2.6] it is assumed that $\left\|A_{0}\right\|_{H_{n-1-\varepsilon}^{\infty}}$ or $\left\|A_{0}\right\|_{H_{n-1}^{\infty}}$ is sufficiently small, respectively. Note also that, in Corollary 6(c), we obtain $f \in \mathcal{B}^{\frac{1}{2}-\varepsilon}$, whereas in [13, Theorems 2.1 and 2.6] it is obtained that $f$ lies in a strictly larger $Q_{K}$ space and some assumptions on $K$ are needed.

Using Corollary 4 and [3, Theorem 5.1], we also find that if (9) holds with $\alpha \in(0,1)$, then $f\left(e^{i t}\right) \in \Lambda_{1-\alpha}$, that is, the boundary function satisfies the Lipschitz condition of order $1-\alpha$. In particular, $f$ belongs to the disc algebra $\mathcal{A}$. Therefore, if the assumption of Corollary 6(b) or 6(c) holds, we get $f \in Q_{K, 0} \cap \mathcal{A}$ or $f \in Q_{K} \cap \mathcal{A}$, respectively. One may now ask whether the solutions could be analytically continued to $\partial \mathbf{D}$ if the coefficients of (1) grow slowly and have a nice boundary behavior. This property is not true in general, as the following counterexample shows.

Example 7. The function $f_{0}(z)=4+2 z+\sum_{k=1}^{\infty} 2^{-k^{2}} z^{2^{k}}$ is one-to-one and continuous in $\overline{\mathbf{D}}$, analytic in $\mathbf{D}$, and all of the derivatives of $f_{0}$ converge uniformly in $\overline{\mathbf{D}}$, see [16, p. 252]. Since $\left|f_{0}(z)\right| \geq 1$ uniformly in $\overline{\mathbf{D}}$, we see that

$$
A(z):=\frac{-f_{0}^{\prime \prime}-f_{0}^{\prime}}{f_{0}}
$$

is analytic in $\mathbf{D}$ and, in fact, belongs to $\mathcal{A}$. In other words, $f_{0} \in \mathcal{A}$ is a solution of

$$
f^{\prime \prime}+f^{\prime}+A(z) f=0
$$

with coefficients in $\mathcal{A}$. Even so, $f_{0}$ cannot be analytically continued to any point of $\partial \mathbf{D}$.

The last result of this section gives a sufficient condition for solutions of (2) to be in $\mathcal{B}^{\alpha}$. This time the condition is given by limiting the Maclaurin coefficients of $A(z)$. 
Corollary 8. Let $f$ be a solution of the equation (2), where $A(z)=\sum_{k=0}^{\infty} a_{k} z^{k} \in$ $\mathcal{H}(\mathbf{D})$. Then the following assertions hold:

(a) If $\alpha \in(0,1)$ and $\left|a_{k}\right|<\alpha(1-\alpha) \frac{\Gamma(k+\alpha+1)}{k ! \Gamma(\alpha+1)}$ for $k \in \mathbf{N} \cup\{0\}$, then $f \in \mathcal{B}^{\alpha}$.

(b) If $\left|a_{k}\right|<\frac{1}{k !} \int_{1}^{2} \frac{\Gamma(k+x)}{\Gamma(x)} d x$ for $k \in \mathbf{N} \cup\{0\}$, then $f \in \mathcal{B}$.

(c) If $\alpha \in(1, \infty)$ and $\left|a_{k}\right|<\alpha(\alpha-1)(1+k)$ for $k \in \mathbf{N} \cup\{0\}$, then $f \in \mathcal{B}^{\alpha}$.

Proof. Since

$$
\frac{1}{(1-z)^{x}}=\sum_{k=0}^{\infty} \frac{\Gamma(k+x)}{k ! \Gamma(x)} z^{k}, \quad z \in \mathbf{D}, \quad x \in(0, \infty),
$$

we obtain

$$
\frac{z}{(1-z)^{2} \log \left(\frac{1}{1-z}\right)}=\int_{1}^{2} \frac{d x}{(1-z)^{x}}=\sum_{k=0}^{\infty} \frac{1}{k !} \int_{1}^{2} \frac{\Gamma(k+x)}{\Gamma(x)} d x z^{k} .
$$

Hence the assumption of the case (a) yields

$$
\begin{aligned}
\sup _{z \in \mathbf{D}}|A(z)|(1-|z|)^{\alpha+1} \int_{0}^{|z|} \frac{d r}{(1-r)^{\alpha}} & \leq \sup _{z \in \mathbf{D}}\left[|A(z)| \frac{(1-|z|)^{\alpha+1}}{1-\alpha}\right] \\
& <\sup _{z \in \mathbf{D}}\left[\alpha(1-|z|)^{\alpha+1} \sum_{k=0}^{\infty} \frac{\Gamma(k+\alpha+1)}{k ! \Gamma(\alpha+1)}|z|^{k}\right]=\alpha,
\end{aligned}
$$

and so the assertion (a) follows by Corollary 4. The assertions (b) and (c) can be proved in a similar manner by using the Maclaurin series above.

Corollary 8 (a) partially improves [13, Theorem 2.4] because there exists $\alpha \in\left(0, \frac{1}{2}\right)$ such that $\alpha(1-\alpha) \frac{\Gamma(k+\alpha+1)}{k ! \Gamma(\alpha+1)}>1$ for $k \geq 12$. Namely, in Corollary $8($ a), we obtain $f \in \mathcal{B}^{\alpha}$, whereas in [13, Theorem 2.4] the condition $\left|a_{k}\right| \leq 1$, for $k \in \mathbf{N}$, gives that $f$ lies in a strictly larger space $\mathcal{D}$. In fact, the assumptions in Corollary 8 allow $\left|a_{k}\right| \rightarrow \infty$ as $k \rightarrow \infty$. This can be seen from the asymptotic estimates

$$
\lim _{k \rightarrow \infty} \frac{\frac{\Gamma(k+\alpha+1)}{k !}}{k^{\alpha}}=1 \quad \text { and } \quad \lim _{k \rightarrow \infty} \frac{\frac{1}{k !} \int_{1}^{2} \frac{\Gamma(k+x)}{\Gamma(x)} d x}{k(\log k)^{-1}}=1,
$$

which are obtained by applying Stirling's approximation.

\section{Polynomial coefficients}

This section contains a straightforward proof of a part of [12, Theorem 8.3], referred to here as Theorem A. In the literature, one can find more technical proofs based on, for example, Wiman-Valiron theory [12] and Herold's comparison theorem [8].

Theorem A. Let the coefficients $A_{0}(z), \ldots, A_{n-1}(z)$ of $(1)$ be polynomials and $A_{n}(z)$ an entire function with a finite order of growth. Then all solutions of (1) are entire functions of finite order. Moreover,

$$
\sigma(f) \leq \max \left\{1+\max _{0 \leq j \leq n-1} \frac{\operatorname{deg}\left(A_{j}\right)}{n-j}, \sigma\left(A_{n}\right)\right\}
$$

for every solution $f$. 
It is a well-known fact that Theorem A is sharp. In fact, for every equation there is a solution for which equality in (13) holds, as is shown in [6, Lemma 3.1].

Before the proof of Theorem A, we note that the end of this section also contains an analogue of Theorem A for the $k$-order of the growth of solutions.

Proof of Theorem A. Assume first that $\sigma\left(A_{n}\right)=0$ and let

$$
\alpha>1+\max _{0 \leq j \leq n-1} \frac{\operatorname{deg}\left(A_{j}\right)}{n-j}
$$

be arbitrary. Define $\omega: \mathbf{C} \rightarrow(0, \infty)$ by $\omega(z)=\exp \left(-(|z|+R)^{\alpha}\right)$ with $R \in(0, \infty)$ to be specified later. Then, in Theorem 2 , we have

$$
\begin{aligned}
I_{m, \omega}(z) & \leq 2^{n} \int_{0}^{z} \int_{0}^{\xi_{1}} \cdots \int_{0}^{\xi_{m-1}} \sum_{j=1}^{m}\left|A_{n-j}^{(m-j)}\left(\xi_{m}\right)\right| e^{\left(\left|\xi_{m}\right|+R\right)^{\alpha}}\left|d \xi_{m}\right| \cdots\left|d \xi_{1}\right| \\
& \leq \frac{2^{n}}{\alpha^{m}} \sup _{|w| \leq|z|} \sum_{j=1}^{m} \frac{\left|A_{n-j}^{(m-j)}(w)\right|}{(|w|+R)^{m(\alpha-1)}} e^{(|z|+R)^{\alpha}}, \quad z \in D .
\end{aligned}
$$

Here the first inequality follows from the estimate $\left(\begin{array}{c}n-j \\ m-j\end{array}\right) \leq \sum_{j=0}^{n}\left(\begin{array}{c}n \\ j\end{array}\right)=2^{n}$. The second one is valid because, for $a: \mathbf{C} \rightarrow[0, \infty)$ and $b:[0, \infty) \rightarrow[0, \infty)$ such that $b^{\prime}$ is non-negative and nondecreasing,

$$
\int_{0}^{z} a(w) e^{b(|w|)}|d w|=\int_{0}^{z} \frac{a(w)}{b^{\prime}(|w|)} b^{\prime}(|w|) e^{b(|w|)}|d w| \leq \sup _{|w| \leq|z|} \frac{a(w)}{b^{\prime}(|w|)} e^{b(|z|)}, \quad z \in \mathbf{C},
$$

which generalizes to

$$
\int_{0}^{z} \int_{0}^{\xi_{1}} \cdots \int_{0}^{\xi_{m-1}} a\left(\xi_{m}\right) e^{b\left(\left|\xi_{m}\right|\right)}\left|d \xi_{m}\right| \cdots\left|d \xi_{1}\right| \leq \sup _{|w| \leq|z|} \frac{a(w)}{b^{\prime}(|w|)^{m}} e^{b(|z|)}, \quad z \in \mathbf{C} .
$$

Now, in Theorem 2, we have

$$
E \leq \sup _{w \in \mathbf{C}} \sum_{m=1}^{n} \frac{2^{n}}{\alpha^{m}} \sum_{j=1}^{m} \frac{\left|A_{n-j}^{(m-j)}(w)\right|}{(|w|+R)^{m(\alpha-1)}}
$$

and hence, by using the fact that $m(\alpha-1)>\operatorname{deg}\left(A_{n-m}\right) \geq \operatorname{deg}\left(A_{n-m}^{(n-j)}\right)$ for all $m=1, \ldots, n$, we can find $R$ such that $E<1$. Therefore Theorem 2 yields that every solution $f$ of (1) satisfies

$$
\sup _{z \in \mathbf{C}}|f(z)| \exp \left(-(|z|+R)^{\alpha}\right)<\infty
$$

and so the assertion follows.

Let $\sigma\left(A_{n}\right) \in(0, \infty)$ and let $\alpha>\sigma\left(A_{n}\right)$ be arbitrary. Since trivially

$$
\left|\int_{0}^{z} \int_{0}^{\xi_{1}} \cdots \int_{0}^{\xi_{n-1}} A_{n}\left(\xi_{n}\right) d \xi_{n} \cdots d \xi_{1}\right| \leq \exp \left((|z|+R)^{\alpha}\right), \quad z \in \mathbf{C},
$$

for sufficiently large $R$, the assertion follows in a similar manner as in the case above.

Now we turn our attention to the $k$-order of non-constant entire functions $f$ defined by

$$
\sigma_{k}(f):=\limsup _{r \rightarrow \infty} \frac{\log _{k+1} M(r, f)}{\log r}, \quad k \in \mathbf{N} .
$$


Here $\log _{1} x=\log x$ and $\log _{k+1} x=\log \left(\log _{k} x\right)$ for sufficiently large $x \in(0, \infty)$. In particular, our purpose is to notice that [1, Theorems 4(i) and 4(ii)], which is an analogue of Theorem A for the $k$-order of the growth of solutions, can be proved by a similar deduction as in Theorem A. Namely, if $A_{j}(z)$ with $j=0, \ldots, n$ are entire functions and

$$
\alpha>\max \left\{\max _{0 \leq j \leq n-1} \sigma_{k}\left(A_{j}\right), \sigma_{k+1}\left(A_{n}\right)\right\}
$$

for some $k \in \mathbf{N}$, then, by proceeding as in the proof of Theorem A with the choice $\omega(z)=1 / \exp _{k+1}\left((|z|+R)^{\alpha}\right)$, we see that every solution of (1) satisfies

$$
\sigma_{k+1}(f) \leq \max \left\{\max _{0 \leq j \leq n-1} \sigma_{k}\left(A_{j}\right), \sigma_{k+1}\left(A_{n}\right)\right\} .
$$

Here $\exp _{1} x=\exp x$ and $\exp _{k+1} x=\exp \left(\exp _{k} x\right)$ for $x \in(0, \infty)$.

\section{Proof of Theorem 1}

We begin this section by stating and proving three lemmas. These lemmas will then be used to prove Theorem 1 . Recall that we use the notation $\omega_{p}(z)=\omega(z)(1-$ $|z|)^{p}$ for $p \in(0, \infty)$ and $z \in \mathbf{D}$.

Lemma 9. Let $\omega: \mathbf{D} \rightarrow(0, \infty)$ be a radial weight satisfying (3). Then, for $f \in \mathcal{H}(\mathbf{D})$,

$$
|f(z)| \omega(z) \leq P_{n} \sup _{|\xi| \leq|z|}\left[\left|f^{(n)}(\xi)\right| \omega(\xi)(1-|\xi|)^{n}\right]+C, \quad z \in \mathbf{D} .
$$

Here $C \in[0, \infty)$ is independent of $z$ and $P_{n}=\prod_{k=1}^{n} M_{k}$ with constants $M_{k}$ as in (5).

Proof. Note first that the condition (3) implies (5) with some constants $M_{k} \leq M$. This follows directly from the inequality $(1-s)^{-k} \leq(1-s)^{-1}(1-r)^{-(k-1)}$ for $s \in[0, r]$ and $k \in \mathbf{N}$.

If $R=R(\omega, M) \in[0,1)$ is close enough to one, then (3) yields

$$
\begin{aligned}
|f(z)| \omega(z) & \leq \int_{0}^{z} \frac{\left|f^{\prime}(\xi)\right| \omega_{1}(\xi)}{\omega_{1}(\xi)}|d \xi| \omega(z)+|f(0)| \omega(z) \\
& \leq \sup _{|\xi| \leq|z|}\left|f^{\prime}(\xi)\right| \omega_{1}(\xi) \int_{0}^{|z|} \frac{d r}{\omega_{1}(r)} \omega(z)+|f(0)| \omega(z) \\
& \leq M \sup _{|\xi| \leq|z|}\left|f^{\prime}(\xi)\right| \omega_{1}(\xi)+|f(0)| \omega(z), \quad R<|z|<1,
\end{aligned}
$$

where the path of integration is the line segment from 0 to $z$. On the other hand, since $\omega$ is bounded, there exists a constant $C^{\prime}=C^{\prime}(\omega, f, R) \geq 0$ such that $|f(z)| \omega(z)<C^{\prime}$ for $|z| \leq R$. Hence (14) holds in the case $n=1$.

Next we assume that (14) holds for $n=N \in \mathbf{N}$. Then

$$
\begin{aligned}
|f(z)| \omega(z) & \leq P_{N} \sup _{|\xi| \leq|z|}\left[\left(\int_{0}^{\xi} \frac{\left|f^{(N+1)}(v)\right| \omega_{N+1}(v)}{\omega_{N+1}(v)}|d v|+\left|f^{(N)}(0)\right|\right) \omega_{N}(\xi)\right]+C_{N} \\
& \leq P_{N} \sup _{|\xi| \leq|z|}\left[\sup _{|v| \leq|\xi|}\left[\left|f^{(N+1)}(v)\right| \omega_{N+1}(v)\right] \int_{0}^{|\xi|} \frac{d r}{\omega_{N+1}(r)} \omega_{N}(\xi)\right]+C_{N+1}^{\prime} \\
& \leq P_{N+1} \sup _{|\xi| \leq|z|}\left[\left|f^{(N+1)}(\xi)\right| \omega_{N+1}(\xi)\right]+C_{N+1},
\end{aligned}
$$

and therefore (14) holds for $n=N+1$. Now the assertion follows by mathematical induction. 
Note that a result similar to Lemma 9 can be obtained without induction by using the formula

$$
f(z)=\int_{0}^{z} \int_{0}^{\xi_{1}} \cdots \int_{0}^{\xi_{n-1}} f^{(n)}\left(\xi_{n}\right) d \xi_{n} \cdots d \xi_{1}+\sum_{j=0}^{n-1} \frac{f^{j}(0)}{j !} z^{j} .
$$

However, in this case, the constant $P_{n}$ may not be the best possible, depending on the behavior of the weight $\omega$ inside the unit disc.

We now proceed to prove the second lemma needed in the proof of Theorem 1 , essentially reversing the estimate obtained in Lemma 9.

Lemma 10. Let $\omega: \mathbf{D} \rightarrow(0, \infty)$ be a radial weight satisfying (4) for some $\varepsilon \in(0, \infty)$ and $m=m(\omega, \varepsilon) \in(0, \infty)$. Then, for $f \in \mathcal{H}(\mathbf{D})$,

$$
\left|f^{(n)}(z)\right| \omega(z)(1-|z|)^{n} \leq n !(1+\varepsilon)^{n} m \sup _{|\xi|=\rho}|f(\xi)| \omega(\rho)+C, \quad z \in \mathbf{D}, \quad n \in \mathbf{N}
$$

where $\rho=\rho(\varepsilon,|z|)=(1+\varepsilon|z|) /(1+\varepsilon)$ and $C \geq 0$ is independent of $z$.

Proof. Since

$$
\frac{\rho}{\rho^{2}-|z|^{2}} \leq \frac{1}{\rho-|z|}=\frac{1+\varepsilon}{1-|z|}
$$

Cauchy's integral formula yields

$$
\left|f^{(n)}(z)\right| \leq n !(1+\varepsilon)^{n} \sup _{|\xi|=\rho}|f(\xi)|(1-|z|)^{-n}, \quad z \in \mathbf{D} .
$$

Hence, by (4), we find $R=R(\omega, \varepsilon, m) \in(0,1)$ such that

$$
\left|f^{(n)}(z)\right| \omega(z)(1-|z|)^{n} \leq n !(1+\varepsilon)^{n} m \sup _{|\xi|=\rho}|f(\xi)| \omega(\rho), \quad R \leq|z|<1 .
$$

Moreover, there exists $C=C(\omega, f, n, R) \in(0, \infty)$ such that $\left|f^{(n)}(z)\right| \omega(z)(1-|z|)^{n} \leq$ $C$ for $|z|<R$. Therefore (15) holds, and the assertion follows.

For future use, we define the dilatation function $f_{r}(z)=f(r z)$, where $z \in \mathbf{D}$ and $r \in[0,1)$.

Lemma 11. Let $\omega: \mathbf{D} \rightarrow(0, \infty)$ be a radial weight such that (4) holds for some $\varepsilon \in(0, \infty)$ and $m=m(\omega, \varepsilon) \in(0, \infty)$. If $f \in \mathcal{H}(\mathbf{D})$ satisfies $\sup _{r \in[0,1)}\left\|f_{r}\right\|_{H_{\omega}^{\infty}}<\infty$, then $f \in H_{\omega}^{\infty}$ and $\|f\|_{H_{\omega}^{\infty}}=\sup _{r \in[0,1)}\left\|f_{r}\right\|_{H_{\omega}^{\infty}}$.

Proof. Assume first that $f \notin H_{\omega}^{\infty}$. Then, for each $n \in \mathbf{N}$, we may choose $z_{n} \in \mathbf{D}$ with $\left|z_{n}\right|>1-\frac{1}{n}$ such that $\left|f\left(z_{n}\right)\right| \omega\left(z_{n}\right)>n$. Let $r_{n}=\left|z_{n}\right|(1+\varepsilon) /\left(1+\varepsilon\left|z_{n}\right|\right)$. Then, by (4),

$$
\begin{aligned}
\left\|f_{r_{n}}\right\|_{H_{\omega}^{\infty}} & \geq\left|f\left(r_{n} \xi_{n}\right)\right| \omega\left(\xi_{n}\right)=\left|f\left(z_{n}\right)\right| \omega\left(z_{n} \frac{1}{\left|z_{n}\right|} \frac{1+\varepsilon\left|z_{n}\right|}{1+\varepsilon}\right) \\
& >\frac{n}{\omega\left(z_{n}\right)} \omega\left(\frac{1+\varepsilon\left|z_{n}\right|}{1+\varepsilon}\right) \geq n \frac{1}{m} \longrightarrow \infty, \quad \xi_{n}=\frac{z_{n}}{r_{n}}
\end{aligned}
$$

as $n \rightarrow \infty$. This is a contradiction, and hence $f \in H_{\omega}^{\infty}$.

Since $M(t, f)=\sup _{\theta \in[0,2 \pi]}\left|f\left(t e^{i \theta}\right)\right|$ is a nondecreasing function of $t$, we have $\sup _{r \in[0,1)}\left\|f_{r}\right\|_{H_{\omega}^{\infty}} \leq\|f\|_{H_{\omega}^{\infty}}$. The converse inequality follows from the definition of supremum and continuity of $f$. 
Proof of Theorem 1. Without loss of generality, assume that $A_{n} \equiv 0$.

(a) If $f$ is a solution of (1), then

$$
f_{r}^{(n)}(z)+\sum_{k=0}^{n-1} B_{k}(z) f_{r}^{(k)}(z)=0, \quad z \in \mathbf{D}
$$

where $B_{j}(z)=B_{j}(z, r)=r^{n-j} A_{j}(r z)$. Since $f_{r} \in H_{\omega}^{\infty}$ for $r \in[0,1)$, Lemma 9 , the equation (16) and Lemma 10 yield

$$
\begin{aligned}
& \left|f_{r}(z)\right| \omega(z) \leq P_{n} \sup _{|\xi| \leq|z|}\left[\left|f_{r}^{(n)}(\xi)\right| \omega_{n}(\xi)\right]+C_{n} \\
& \leq P_{n} \sup _{|\xi| \leq|z|}\left[\sum_{k=0}^{n-1}\left|B_{k}(\xi)\right|(1-|\xi|)^{n-k}\left|f_{r}^{(k)}(\xi)\right| \omega_{k}(\xi)\right]+C_{n} \\
& \leq P_{n}\left[\left\|B_{0}\right\|_{H_{n-k}^{\infty}}\left\|f_{r}\right\|_{H_{\omega}^{\infty}}+\sum_{k=1}^{n-1}\left\|B_{k}\right\|_{H_{n-k}^{\infty}}\left(k !(1+\varepsilon)^{k} m \sup _{|\xi|=\rho}\left|f_{r}(\xi)\right| \omega(\rho)+C_{k}\right)\right]+C_{n} \\
& \leq E\left\|f_{r}\right\|_{H_{\omega}^{\infty}}+C,
\end{aligned}
$$

where the constants $C, C_{j} \in(0, \infty)$ are independent of $z$ for $j=0,1, \ldots, n$. Hence

$$
\sup _{r \in[0,1)}\left\|f_{r}\right\|_{H_{\omega}^{\infty}} \leq \frac{C}{1-E}<\infty
$$

and consequently $f \in H_{\omega}^{\infty}$ by Lemma 11 .

(b) Similarly as in Lemma 9, we have

$$
|f(z)| \omega(z) \leq \sup _{|\xi| \leq|z|}\left|f^{\prime}(\xi)\right| \omega(\xi) \int_{0}^{|z|} \frac{d r}{\omega(r)} \omega(z)+|f(0)| \omega(z), \quad z \in \mathbf{D},
$$

for $f \in \mathcal{H}(\mathbf{D})$. Moreover, by applying Lemma 9 for $f^{\prime}$ and $n-1$ instead of $f$ and $n$, we obtain

$$
\left|f^{\prime}(z)\right| \omega(z) \leq P_{n-1} \sup _{|\xi| \leq|z|}\left[\left|f^{(n)}(\xi)\right| \omega_{n-1}(\xi)\right]+C .
$$

Hence, the conditions (18), (1) and (17) yield

$$
\left|f_{r}^{\prime}(z)\right| \omega(z) \leq F\left\|f_{r}^{\prime}\right\|_{H_{\omega}^{\infty}}+C,
$$

where the constant $C \in(0, \infty)$ is independent of $z$. Now the assertion $f^{\prime} \in H_{\omega}^{\infty}$ follows by Lemma 11.

In the cases

$$
f^{(n)}+A_{0}(z) f=0 \quad \text { and } \quad f^{(n)}+A_{1}(z) f^{\prime}+A_{0}(z) f=0
$$

for assertions (a) and (b), respectively, the estimate of Lemma 10 is not needed, and hence the proofs above may be written directly for $f$ instead of the dilatation $f_{r}$. Thus we also do not need Lemma 11 and consequently the assumption (4) regarding the weight $\omega$ is not necessary.

\section{Proof of Theorem 2}

This section contains the proof of Theorem 2. Before the proof, we state the following lemma which is a simple consequence of Leibniz's rule and mathematical induction. 
Lemma 12. If $f, g \in \mathcal{H}(D)$, then

$$
f^{(n)}(z) g(z)=\sum_{j=0}^{n}(-1)^{j}\left(\begin{array}{l}
n \\
j
\end{array}\right)\left(f g^{(j)}\right)^{(n-j)}(z), \quad z \in D
$$

for any $n \in \mathbf{N}$.

In order to simplify some of the formulas in the following proof, we use the interpretation $\sum_{j=0}^{-1}(\cdot)=0$, that is, a sum, whose starting value of the summation index is higher than the end value, has no summands.

Proof of Theorem 2. (a) If $f$ is a solution of (1), then, by applying the identity

$$
f(z)=\int_{0}^{z} f^{\prime}(\xi) d \xi+f(0), \quad z \in D
$$

$n$ times and using equation (1) and Lemma 12, we obtain

$$
\begin{aligned}
& |f(z)| \omega(z) \\
& \leq\left|\int_{0}^{z} \int_{0}^{\xi_{1}} \cdots \int_{0}^{\xi_{n-1}}\left[A_{n-1}\left(\xi_{n}\right) f^{(n-1)}\left(\xi_{n}\right)+\ldots+A_{0}\left(\xi_{n}\right) f\left(\xi_{n}\right)\right] d \xi_{n} \cdots d \xi_{1}\right| \omega(z)+C_{1} \\
& =\left|\int_{0}^{z} \int_{0}^{\xi_{1}} \cdots \int_{0}^{\xi_{n-1}} \sum_{k=0}^{n-1} \sum_{j=0}^{k}(-1)^{j}\left(\begin{array}{c}
k \\
j
\end{array}\right)\left(A_{k}^{(j)} f\right)^{(k-j)}\left(\xi_{n}\right) d \xi_{n} \cdots d \xi_{1}\right| \omega(z)+C_{1},
\end{aligned}
$$

where

$C_{1}=\sup _{z \in D} \omega(z) \sum_{j=0}^{n-1} \frac{\left|f^{(j)}(0)\right|}{j !}+\|g\|_{H_{\omega}^{\infty}(D)}, \quad g(z)=\int_{0}^{z} \int_{0}^{\xi_{1}} \cdots \int_{0}^{\xi_{n-1}} A_{n}\left(\xi_{n}\right) d \xi_{n} \cdots d \xi_{1}$.

Since

$$
\begin{aligned}
\int_{0}^{z} \int_{0}^{\xi_{1}} \cdots \int_{0}^{\xi_{n-1}} \sum_{k=0}^{n-1} \sum_{j=0}^{k}(-1)^{j}\left(\begin{array}{c}
k \\
j
\end{array}\right)\left(A_{k}^{(j)} f\right)^{(k-j)}\left(\xi_{n}\right) d \xi_{n} \cdots d \xi_{1} \\
=\sum_{k=0}^{n-1} \sum_{j=0}^{k}(-1)^{j}\left(\begin{array}{c}
k \\
j
\end{array}\right) \int_{0}^{z} \int_{0}^{\xi_{1}} \cdots \int_{0}^{\xi_{n-1-(k-j)}}\left[A_{k}^{(j)}\left(\xi_{n-(k-j)}\right) f\left(\xi_{n-(k-j)}\right)\right. \\
\left.\quad-\sum_{l=0}^{k-j-1} \frac{\left(A_{k}^{(j)} f\right)^{(l)}(0)}{l !} \xi_{n-(k-j)}^{l}\right] d \xi_{n-(k-j)} \cdots d \xi_{1} \\
=\sum_{m=1}^{n} \int_{0}^{z} \int_{0}^{\xi_{1}} \cdots \int_{0}^{\xi_{m-1}}\left[\sum_{j=1}^{m}(-1)^{m-j}\left(\begin{array}{c}
n-j \\
m-j
\end{array}\right) A_{n-j}^{(m-j)}\left(\xi_{m}\right)\right] f\left(\xi_{m}\right) d \xi_{m} \cdots d \xi_{1} \\
\quad-\sum_{k=0}^{n-1} \sum_{j=0}^{k} \sum_{l=0}^{k-j-1}(-1)^{j}\left(\begin{array}{c}
k \\
j
\end{array}\right) \frac{\left(A_{k}^{(j)} f\right)^{(l)}(0)}{(n-k+j+l) !} z^{n-k+j+l},
\end{aligned}
$$

we have

$$
|f(z)| \omega(z) \leq \sup _{\xi \in[0, z]}|f(\xi)| \omega(\xi) \sup _{\xi \in D} \omega(\xi) \sum_{m=1}^{n} I_{m, \omega}(\xi)+C, \quad z \in D,
$$

where $[0, z]$ is the closed line segment from 0 to $z$. 
Let now $K$ be a compact subset of $D$ containing the line segment $[0, z]$ for all $z \in K$. Then the formula above together with (7) and the estimate

$$
\sup _{\xi \in[0, z]}|f(\xi)| \omega(\xi) \leq \sup _{\xi \in K}|f(\xi)| \omega(\xi), \quad z \in K
$$

yield

$$
\sup _{\xi \in K}|f(\xi)| \omega(\xi) \leq \frac{C}{1-E}<\infty
$$

Since this holds for all compact sets $K$ with the properties mentioned above, we obtain $\|f\|_{H_{\omega}^{\infty}(D)} \leq \frac{C}{1-E}$, which completes the proof of assertion (a).

(b) Similarly as in the proof of part (a), we obtain

$$
\begin{aligned}
& \left|f^{\prime}(z)\right| \omega(z) \\
& \leq\left|\int_{0}^{z} \cdots \int_{0}^{\xi_{n-2}} \sum_{k=1}^{n-1} \sum_{j=0}^{k-1}(-1)^{j}\left(\begin{array}{c}
k-1 \\
j
\end{array}\right)\left(A_{k}^{(j)} f^{\prime}\right)^{(k-1-j)}\left(\xi_{n-1}\right) d \xi_{n-1} \cdots d \xi_{1}\right| \omega(z) \\
& \quad+\left|\int_{0}^{z} \cdots \int_{0}^{\xi_{n-2}} A_{0}\left(\xi_{n-1}\right) f\left(\xi_{n-1}\right) d \xi_{n-1} \cdots d \xi_{1}\right| \omega(z)+C_{1}, \quad z \in D,
\end{aligned}
$$

where

$$
C_{1}=\sup _{z \in D} \omega(z) \sum_{j=0}^{n-2} \frac{\left|f^{(j+1)}(0)\right|}{j !}+\|g\|_{H_{\omega}^{\infty}(D)}, \quad g(z)=\int_{0}^{z} \cdots \int_{0}^{\xi_{n-2}} A_{n}\left(\xi_{n-1}\right) d \xi_{n-1} \cdots d \xi_{1} .
$$

Since

$$
\begin{aligned}
& \left|\int_{0}^{z} \cdots \int_{0}^{\xi_{n-2}} A_{0}\left(\xi_{n-1}\right) f\left(\xi_{n-1}\right) d \xi_{n-1} \cdots d \xi_{1}\right| \\
& \leq\left[\sup _{\xi \in[0, z]}\left|f^{\prime}(\xi)\right| \omega(\xi)\right] \int_{0}^{z} \cdots \int_{0}^{\xi_{n-2}}\left|A_{0}\left(\xi_{n-1}\right)\right| \int_{0}^{\xi_{n-1}} \frac{\left|d \xi_{n}\right|}{\omega\left(\xi_{n}\right)}\left|d \xi_{n-1}\right| \cdots\left|d \xi_{1}\right| \\
& \quad+|f(0)|\left|\int_{0}^{z} \cdots \int_{0}^{\xi_{n-2}} A_{0}\left(\xi_{n-1}\right) d \xi_{n-1} \cdots d \xi_{1}\right|, \quad z \in D
\end{aligned}
$$

and

$$
\begin{aligned}
& \int_{0}^{z} \cdots \int_{0}^{\xi_{n-2}} \sum_{k=1}^{n-1} \sum_{j=0}^{k-1}(-1)^{j}\left(\begin{array}{c}
k-1 \\
j
\end{array}\right)\left(A_{k}^{(j)} f^{\prime}\right)^{(k-1-j)}\left(\xi_{n-1}\right) d \xi_{n-1} \cdots d \xi_{1} \\
& =\sum_{m=1}^{n-1} \int_{0}^{z} \cdots \int_{0}^{\xi_{m-1}}\left[\sum_{j=1}^{m}(-1)^{m-j}\left(\begin{array}{c}
n-1-j \\
m-j
\end{array}\right) A_{n-j}^{(m-j)}\left(\xi_{m}\right)\right] f^{\prime}\left(\xi_{m}\right) d \xi_{m} \cdots d \xi_{1} \\
& -\sum_{k=1}^{n-1} \sum_{j=0}^{k-1} \sum_{l=0}^{k-j-2}(-1)^{j}\left(\begin{array}{c}
k-1 \\
j
\end{array}\right) \frac{\left(A_{k}^{(j)} f^{\prime}\right)^{(l)}(0)}{(n-k+j+l) !} z^{n-k+j+l}, \quad z \in D,
\end{aligned}
$$

we have

$$
\begin{aligned}
\left|f^{\prime}(z)\right| \omega(z) \leq & \sup _{\xi \in[0, z]}\left|f^{\prime}(\xi)\right| \omega(\xi) \sup _{\xi \in D} \omega(\xi)\left[\sum_{m=1}^{n-1} I_{m, \omega}^{*}(\xi)\right. \\
& \left.+\int_{0}^{\xi} \cdots \int_{0}^{\xi_{n-2}}\left|A_{0}\left(\xi_{n-1}\right)\right| \int_{0}^{\xi_{n-1}} \frac{\left|d \xi_{n}\right|}{\omega\left(\xi_{n}\right)}\left|d \xi_{n-1}\right| \cdots\left|d \xi_{1}\right|\right]+C, \quad z \in D .
\end{aligned}
$$


Here

$$
\begin{aligned}
C= & \sup _{z \in D} \omega(z)\left(\sum_{j=0}^{n-2} \frac{\left|f^{(j+1)}(0)\right|}{j !}+\sum_{k=1}^{n-1} \sum_{j=0}^{k-1} \sum_{l=0}^{k-j-2}\left(\begin{array}{c}
k-1 \\
j
\end{array}\right) \frac{\left|\left(A_{k}^{(j)} f^{\prime}\right)^{(l)}(0)\right|}{(n-k+j+l) !}\right) \\
& +|f(0)| \sup _{z \in D} \omega(z)\left|\int_{0}^{z} \cdots \int_{0}^{\xi_{n-2}} A_{0}\left(\xi_{n-1}\right) d \xi_{n-1} \cdots d \xi_{1}\right|+\|g\|_{H_{\omega}^{\infty}(D)} \\
<\infty &
\end{aligned}
$$

because

$$
\sup _{z \in D} \omega(z) \int_{0}^{z} \cdots \int_{0}^{\xi_{n-2}}\left|A_{0}\left(\xi_{n-1}\right)\right| \int_{0}^{\xi_{n-1}} \frac{\left|d \xi_{n}\right|}{\omega\left(\xi_{n}\right)}\left|d \xi_{n-1}\right| \cdots\left|d \xi_{1}\right|<\infty
$$

by (8), and $\int_{0}^{\xi_{n-1}} \frac{\left|d \xi_{n}\right|}{\omega\left(\xi_{n}\right)}$ is zero only if $\xi_{n-1}=0$. Hence (8) yields

$$
\sup _{\xi \in K}\left|f^{\prime}(\xi)\right| \omega(\xi) \leq \frac{C}{1-F}<\infty
$$

for all compact sets $K \subset D$ containing the line segments $[0, z]$ for $z \in K$, and consequently $f^{\prime} \in H_{\omega}^{\infty}(D)$.

Acknowledgements. The authors thank Janne Heittokangas and Jouni Rättyä for helpful comments and the referees for careful reading of the manuscript and valuable suggestions.

\section{References}

[1] Bernal, L.: On growth $k$-order of solutions of a complex homogeneous linear differential equation. - Proc. Amer. Math. Soc. 101, 1987, 317-322.

[2] Chyzhykov, I., G. Gundersen, and J. Heittokangas: Linear differential equations and logarithmic derivative estimates. - Proc. London Math. Soc. 86, 2003, 735-754.

[3] Duren, P.: Theory of $H^{p}$ spaces. - Academic Press, New York, 1970.

[4] EsSÉn, M., and H. Wulan: On analytic and meromorphic functions and spaces of $Q_{K}$-type. - Illinois J. Math. 46, 2002, 1233-1258.

[5] GröHn, J.: New applications of Picard's successive approximations. - Bull. Sci. Math. 135, $2011,475-487$.

[6] Gundersen, G., E. Steinbart, and S. Wang: Growth and oscillation theory of nonhomogeneous linear differtial equations. - Proc. Edinburgh Math. Soc. 43, 2000, 343-359.

[7] Heittokangas, J.: On complex differential equations in the unit disc. - Ann. Acad. Sci. Fenn. Math. Diss. 122, 2000, 1-54.

[8] Heittokangas, J., R. Korhonen, and J. RÄтtyä: Growth estimates for solutions of linear complex differential equations. - Ann. Acad. Sci. Fenn. Math. 29, 2004, 233-246.

[9] Heittokangas, J., R. Korhonen, and J. RÄтtYä: Fast growing solutions of linear differential equations in the unit disc. - Results Math. 49, 2006, 265-278.

[10] Heittokangas, J., R. Korhonen, and J. Rättyä: Linear differential equations with solutions in the Dirichlet type subspace of the Hardy space. - Nagoya Math. J. 187, 2007, 91-113.

[11] Heittokangas, J., R. Korhonen, and J. RÄTtyÄ: Growth estimates for solutions of nonhomogeneous linear complex differential equations. - Ann. Acad. Sci. Fenn. Math. 34, 2009, $145-156$.

[12] Laine, I.: Nevanlinna theory and complex differential equations. - Walter de Gruyter, Berlin, 1993. 
[13] Li, H., and H. Wulan: Linear differential equations with solutions in the $Q_{K}$ spaces. - J. Math. Anal. Appl. 375, 2011, 478-489.

[14] PelÁez, J., and J. RäTtYä: Weighted Bergman spaces induced by rapidly increasing weights. - Mem. Amer. Math. Soc. 227, 2014.

[15] Pommerenke, Ch.: On the mean growth of the solutions of complex linear differential equations in the disk. Complex Var. Theory Appl. 1, 1982, 23-38.

[16] Remmert, R.: Classical topics in complex function theory. - Springer, Berlin, 1998.

[17] Shields, A., and D. Williams: Bounded projections and the growth of harmonic conjugates in the unit disc. - Michigan Math. J. 29, 1982, 3-25.

[18] Wiтtich, H.: Zur Theorie linearer Differentialgleichungen im Komplexen. - Ann. Acad. Sci. Fenn. Ser. A I Math. 379, 1966, 1-18.

Received 25 September $2014 \bullet$ Revised received 11 September 2014 • Accepted 2 October 2015 